\title{
Supply-Based Dynamic Ramsey Pricing with Two Sectors: Avoiding Water Shortages
}

\author{
Yiğit Sağlam \\ NZISCR and School of Economics and Finance \\ Victoria University of Wellington
}

NZISCR

May 2, 2012 


\section{Introduction}

Two stylized factors in water markets:

- Around ninety percent of all surface water reservoirs are managed by local or federal governments, and running a balanced budget has been a top priority.

- In many parts of the world (including sub-Saharan Africa, Middle East, and Southern Europe), countries suffer from water supply volatility accompanied by temporary but frequent water shortages.

- OECD: "Several OECD countries have experienced periodic water shortages, based on high levels of leakage in the water supply systems, or inefficient usage encountered by insufficient pricing policies." 


\section{Introduction}

1. Agricultural sector:

* About $70 \%$ of all withdrawals in the world are by agriculture.

* Government subsidization towards agricultural sector.

* Non-volumetric irrigation pricing schemes.

2. Water price paid by agriculture is around $1 \%$ of tap price paid by households and industry; see figure 1 :

* The United States: $\$ 0.05$ per $\mathrm{m}^{3}$ vs. $\$ 1.25$ per $\mathrm{m}^{3}$

* France: $\$ 0.08$ per $\mathrm{m}^{3}$ vs. $\$ 3.11$ per $\mathrm{m}^{3}$

* Italy, Japan, Turkey: Non-volumetric irrigation pricing schemes. 


\section{Introduction}

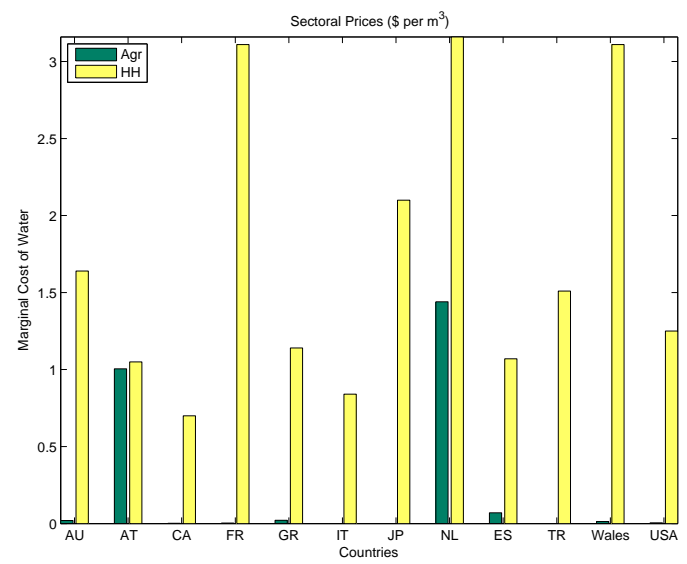

Figure: Water Prices for Different Sectors in OECD Countries 


\section{Research Questions}

1. To what extent an optimal pricing rule can avoid these water shortages?

* Structural estimation of the model using monthly data from Turkey on water flows, crop compositions, water and crop prices, from 1984 to 2007.

* Implications of current and optimal water pricing rules on water management and water users

2. Alternative measures under the ACP rule:

* Supply-side measures: Increasing reservoir capacity, preventing leakages

* Demand-side measures: Lower crop-water requirements 


\section{Key Features}

1. Partial equilibrium model with revenue and resource constraints, and multiple sectors

2. Changes in crop composition in response to water scarcity along with other factors (crop prices, land productivity).

3. The water supplier may charge higher prices. Nonetheless, all profits are rebated back to the consumers and producers.

4. Empirical Analysis: Structural estimation of crop composition and tap water demand, and quantitative comparisons of the two pricing policies

5. Program Evaluation: Monte Carlo Simulations to evaluate the frequency of water shortages. 


\section{Model}

- Partial equilibrium model for water

- Demand for water: Monthly demand by households and seasonal demand by agriculture.

- Supply for water: A benevolent government controls two water prices. 


\section{Households}

- Consumers spend their income on tap water and a composite good.

- Tap water may have different uses, such as drinking (price-non-responsive) and non-drinking (price-responsive) components.

- Utility maximization problem leads to the total demand for tap water. 


\section{Agriculture}

- Producers are identical farmers in a perfectly competitive output market.

- Leontief production function in agriculture depends on land and water.

- Mixed-Choice Problem:

* Farmers choose which crop to produce.

* Having chosen the crop, the farmers then decide how much land to allocate. 


\section{Estimation: Irrigation Water}

- Model: Representative farmer's solution at the equilibrium is true for all farmers. 


\section{Estimation: Irrigation Water}

- Model: Representative farmer's solution at the equilibrium is true for all farmers.

- Data: Heterogeneity in crop choices across farmers and time 


\section{Estimation: Irrigation Water}

- Model: Representative farmer's solution at the equilibrium is true for all farmers.

- Data: Heterogeneity in crop choices across farmers and time

- General Equilibrium: Farmers would be indifferent across crops. 


\section{Estimation: Irrigation Water}

- Model: Representative farmer's solution at the equilibrium is true for all farmers.

- Data: Heterogeneity in crop choices across farmers and time

- General Equilibrium: Farmers would be indifferent across crops.

- Partial Equilibrium with iid shocks across farmers and time 


\section{Agents}

- The government

* observes the total stock at the beginning of each period,

* chooses the two water prices optimally,

* rebates all the profits back as a lump-sum transfer.

- Dynamic Ramsey Pricing Problem is:

* to maximize discounted expected lifetime utility of agents:

* subject to dynamic resource constraint

* subject to sectoral revenue constraints.

- In case of a water shortage, the government uses rationing for both sectors. 


\section{Data}

- Data collection:

* Water flows data from the State Water Works

* Irrigation price and land allocation data from the local water user associations

* Tap price, quantity, and water sanitation data from the municipality

* Climatic variables from Turkish Meteorological Institute

- Monthly time-series data from 01/1984 to 08/2007

- Irrigation prices and land allocation are yearly data from 1984 to 2007 . 


\section{Figure: Location}

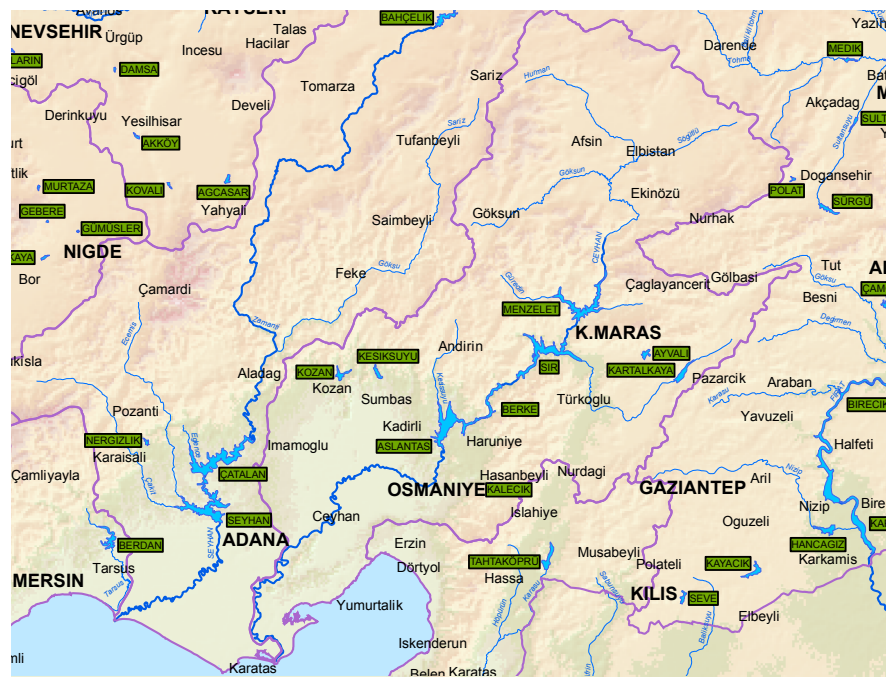

Figure: Geographical (GIS) Map of Cukurova 


\section{Figure: Flows}
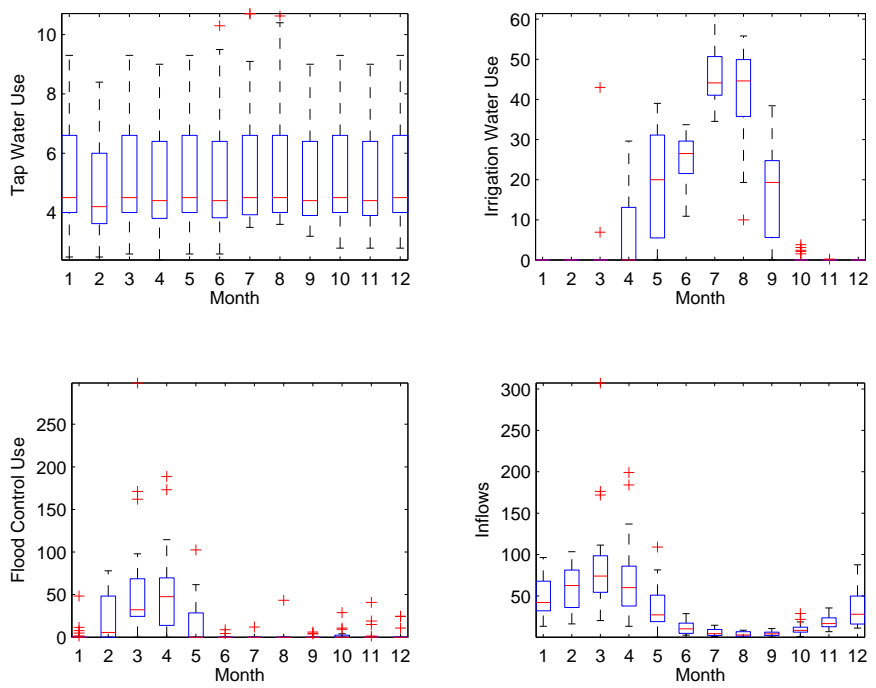

Figure: Reservoir Flows (January-December) 
Figure: Tap Price vs Revenue: Inelastic demand for tap water.

Tap Water $\left(\mathrm{m}^{3}\right)$ vs. Price (per $\left.100 \mathrm{~m}^{3}\right)$

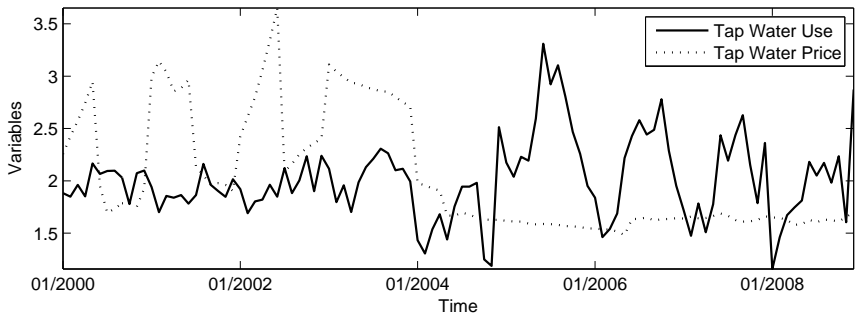

Revenue vs. Price

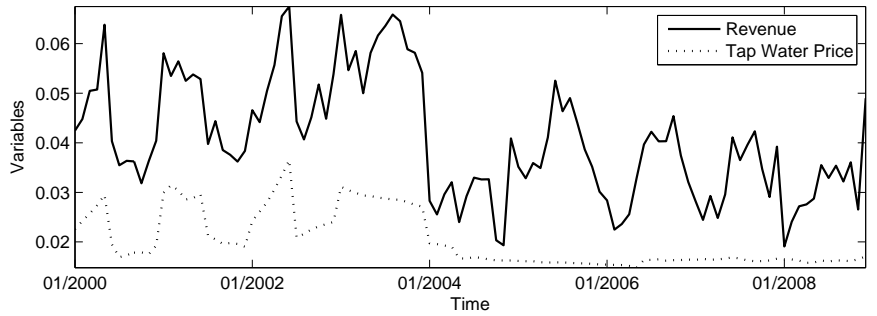

Figure: Tap Water Use and Price 


\section{Figure: Irrigation Prices}

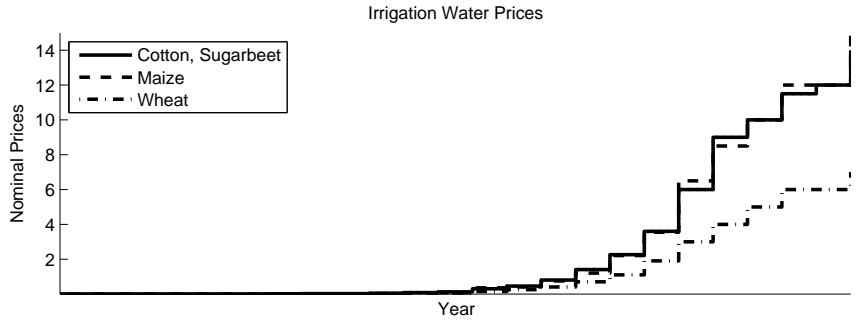

Irrigation Water Real Prices $(1994=100)$

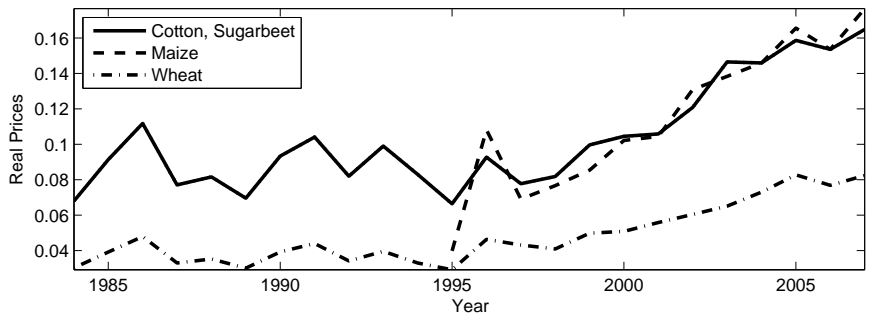

Figure: Irrigation Water Prices 
Figure: Crop Composition
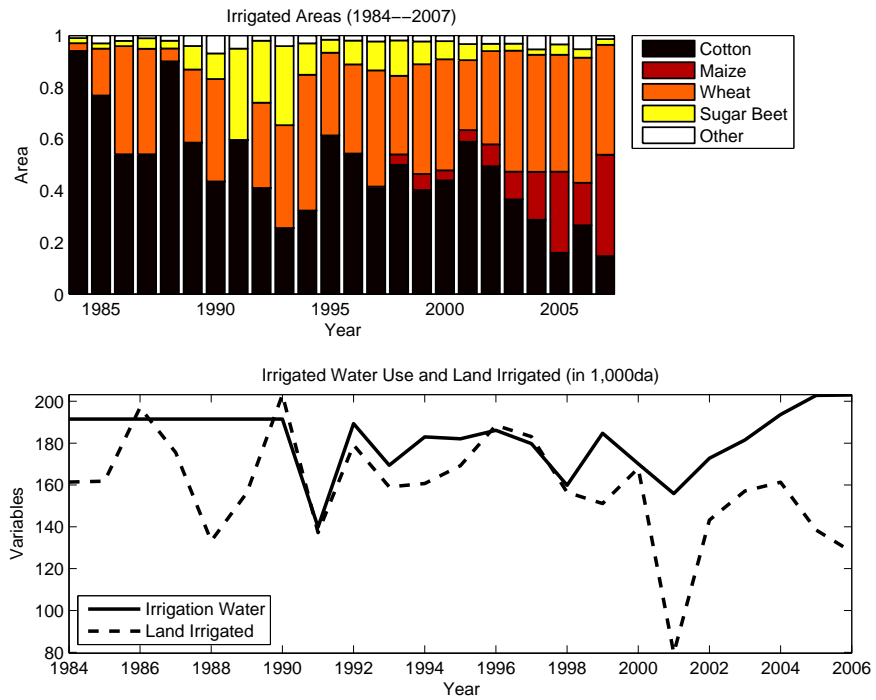

Figure: Crop Composition 


\section{Estimation: Tap Water}

Functional Form for the Tap Water Demand

- is consistent with utility maximization problem

- delivers inelastic demand for tap water.

Stone-Geary functional form for the utility.

$$
U=\pi_{1} \log \left(w_{1}-\underline{w}_{1}\right)+\left(1-\pi_{1}\right) \log y
$$

Demand for tap water is:

$$
w_{1}=\left(1-\pi_{1}\right) \underline{w}_{1}+\pi_{1} \frac{I}{p_{1}}
$$




\section{Estimation: Tap Water}

Demand for tap water is:

$$
w_{1}=\left(1-\pi_{1}\right) \underline{w}_{1}+\pi_{1} \frac{I}{p_{1}}
$$

Parameters to Estimate:

- $\underline{w}_{1}$ : subsistence level

- $\pi_{1}$ : marginal budget of tap water

Methods: Least Absolute Deviation (LAD) vs.Least-Squares (LS) Methods 


\section{Estimation: Tap Water}

\begin{tabular}{|c|c|c|c|c|c|c|c|c|}
\hline & \multicolumn{4}{|c|}{ Stone-Geary } & \multicolumn{4}{|c|}{ Double Log } \\
\hline Variable & \multicolumn{2}{|c|}{ LS } & \multicolumn{2}{|c|}{ LAD } & \multicolumn{2}{|c|}{ LS } & \multicolumn{2}{|c|}{ LAD } \\
\hline Constant & $\begin{array}{r}1.6969 \\
(0.1160)\end{array}$ & $\begin{array}{r}1.8418 \\
(0.1223)\end{array}$ & $\begin{array}{r}1.6875 \\
(0.0708)\end{array}$ & $\begin{array}{r}1.8007 \\
(0.0945)\end{array}$ & $\begin{array}{r}-0.1750 \\
(0.2959)\end{array}$ & $\begin{array}{r}-0.0791 \\
(0.3178)\end{array}$ & $\begin{array}{c}-0.1444 \\
(0.236)\end{array}$ & $\begin{array}{r}-0.2464 \\
(0.3043)\end{array}$ \\
\hline$I / p_{1}$ & $\begin{array}{r}0.0005 \\
(0.0002)\end{array}$ & $\begin{array}{l}0.0005 \\
0.0002\end{array}$ & $\begin{array}{r}0.0006 \\
(0.0001)\end{array}$ & $\begin{array}{r}0.0005 \\
(0.0002)\end{array}$ & - & - & - & - \\
\hline $\log p_{1}$ & - & - & - & - & $\begin{array}{r}0.2517 \\
(0.1022)\end{array}$ & $\begin{array}{r}0.1682 \\
(0.10365)\end{array}$ & $\begin{array}{r}0.2512 \\
(0.0859)\end{array}$ & $\begin{array}{r}0.2322 \\
(0.0924)\end{array}$ \\
\hline $\log I$ & - & - & - & - & $\begin{array}{r}0.7941 \\
(0.1853)\end{array}$ & $\begin{array}{r}0.6335 \\
(0.2058)\end{array}$ & $\begin{array}{r}0.7827 \\
(0.1833)\end{array}$ & $\begin{array}{r}0.8101 \\
(0.1993)\end{array}$ \\
\hline$\theta_{1}$ & - & $\begin{array}{r}-0.0019 \\
(0.0005)\end{array}$ & - & $\begin{array}{r}-0.0009 \\
(0.0006)\end{array}$ & - & $\begin{array}{r}-0.0007 \\
(0.0002)\end{array}$ & - & $\begin{array}{r}-0.0003 \\
(0.0003)\end{array}$ \\
\hline $\log L w_{1}$ & - & - & - & - & - & - & - & - \\
\hline Obs. & 108 & 97 & 108 & 97 & 108 & 97 & 108 & 97 \\
\hline
\end{tabular}

\begin{tabular}{|c|c|c|c|c|}
\hline & \multicolumn{4}{|c|}{ Double Log PA } \\
\hline Variable & \multicolumn{2}{|c|}{ LS } & \multicolumn{2}{|c|}{ LAD } \\
\hline Constant & $\begin{array}{r}-0.1345 \\
(0.2626)\end{array}$ & $\begin{array}{r}0.0898 \\
(0.2796)\end{array}$ & $\begin{array}{r}-0.023 \\
(0.2120))\end{array}$ & $\begin{array}{r}0.3057 \\
(0.2600)\end{array}$ \\
\hline$I / p_{1}$ & - & - & - & - \\
\hline $\log p_{1}$ & $\begin{array}{r}0.1233 \\
(0.0933)\end{array}$ & $\begin{array}{r}0.0424 \\
(0.0935)\end{array}$ & $\begin{array}{r}0.0786 \\
(0.0739)\end{array}$ & $\begin{array}{r}0.0522 \\
(0.0828)\end{array}$ \\
\hline $\log I$ & $\begin{array}{r}0.4173 \\
(0.1775)\end{array}$ & $\begin{array}{r}0.1947 \\
(0.1967)\end{array}$ & $\begin{array}{r}0.2684 \\
(0.1535)\end{array}$ & $\begin{array}{r}0.0793 \\
(0.1823)\end{array}$ \\
\hline$\theta_{1}$ & - & $\begin{array}{r}-0.0007 \\
(0.0002)\end{array}$ & - & $\begin{array}{r}-0.0008 \\
(0.0003)\end{array}$ \\
\hline $\log L w_{1}$ & $\begin{array}{r}0.4879 \\
(0.0875)\end{array}$ & $\begin{array}{r}0.5106 \\
(0.0932)\end{array}$ & $\begin{array}{r}0.5821 \\
(0.0929)\end{array}$ & $\begin{array}{r}0.6447 \\
(0.0919)\end{array}$ \\
\hline Obs. & 107 & 96 & 107 & 96 \\
\hline
\end{tabular}




\section{Estimation: Irrigation Water}

- Leontief production function:

$$
f\left(\ell_{c}, w_{2 c}\right)=\alpha_{c} \ell_{c} \min \left(1, \frac{w_{2 c}}{\gamma_{c}}\right) ; \forall c=1, \ldots, N
$$

- The representative farmer solves a mixed-choice problem:

$$
\begin{aligned}
\Pi & =\max \left(\Pi_{1}, \ldots, \Pi_{N}, \Pi_{N+1}\right) \text { where } \\
\Pi_{c} & =\max _{<\ell_{c}>}\left(p_{f c} f\left(\ell_{c}, w_{2 c}\right)-p_{2} w_{2 c}+\mu_{c} \ell_{c}\right) ; \forall c=1, \ldots, N \\
\ni \ell_{c} & \leq \bar{\ell}=1, \\
\Pi_{N+1} & =0
\end{aligned}
$$




\section{Estimation: Irrigation Water}

- Irrigation Water Demand

* $\left\{\alpha_{c}\right\}_{c=1}^{N}$ : land productivity

* $\left\{\gamma_{c}\right\}_{c=1}^{N}$ : crop water requirements

$*\left\{\mu_{c}\right\}_{c=1}^{N}$ : mean values of shocks

- Method: due to little variation in crop and irrigation prices,

* I calibrate $\alpha$, and $\gamma$-technological parameters,

* I estimate $\mu$ using the generalized method of moments.

\begin{tabular}{rrrrr}
\hline & Cotton & Maize & Wheat & Sugar beets \\
\hline Coefficient & 1.4963 & -2.7698 & 0.7233 & -5.049 \\
Standard Error & 0.1761 & 0.4333 & 0.1818 & 0.4333 \\
Gradient $(\times 1 e-4)$ & 0.0001 & 0 & -0.0001 & 0 \\
Objective $(\times 1 e-6)$ & 0 & & & \\
Number of Observations & 24 & & & \\
\hline
\end{tabular}

Table: Estimation of Land Allocations 


\section{Figure: Irrigation Water Demand}
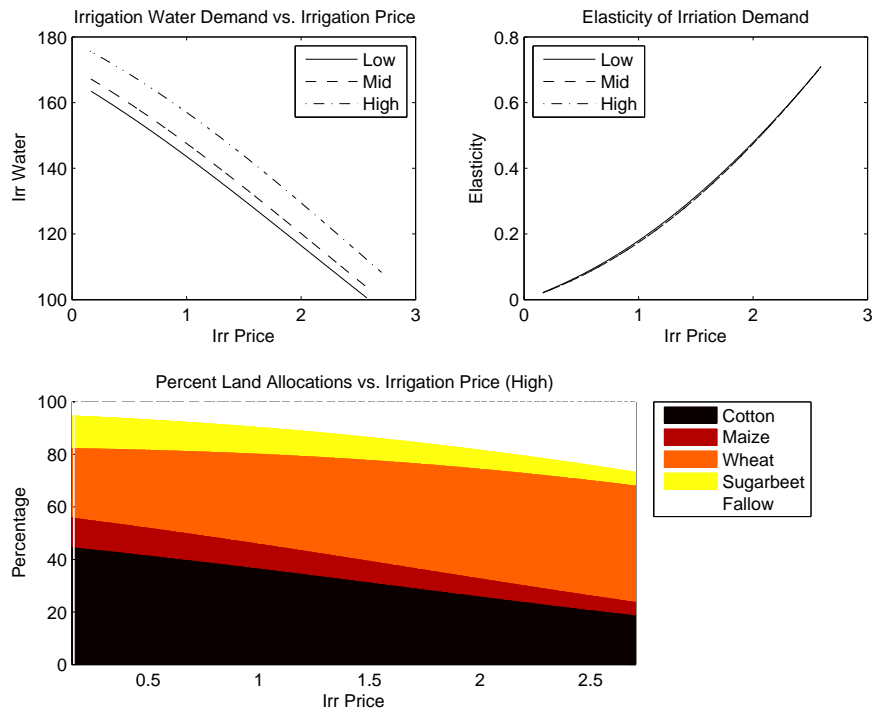

Figure: Irrigation Water Demand 


\section{Figure: Water Shortages}
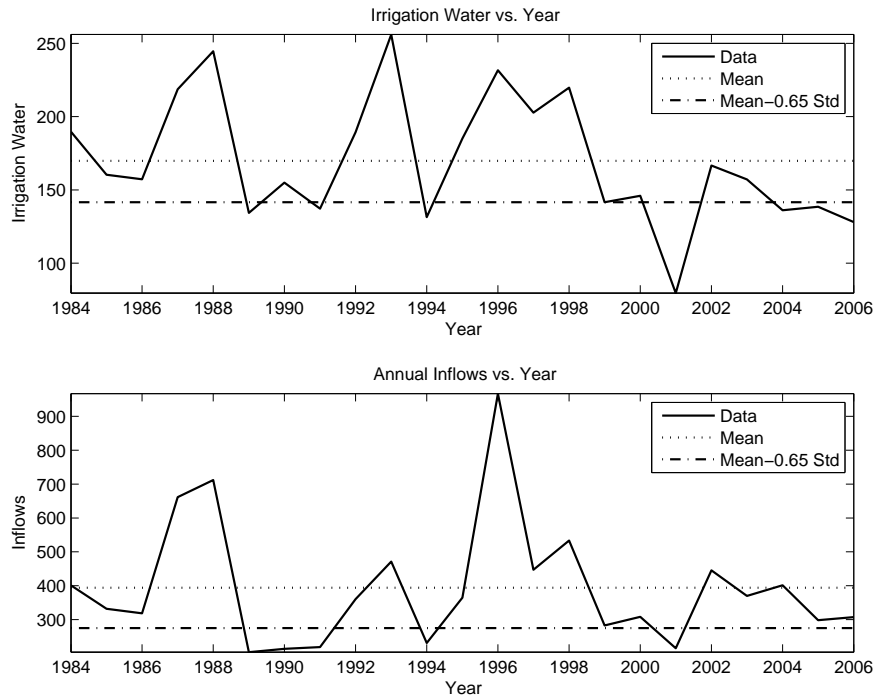

Figure: Water Shortages in the Turkish Data 


\section{Model Fit and Counterfactuals}

Definition: A water shortage when the irrigation water use is less than 0.65 times its standard deviation below the sample mean.

- Questions:

* Starting from 01/1984, can the model predict the years with water shortage?

* Can these water shortages in the last 24 years be avoided using optimal pricing rule?

- Method:

* Assign the state variables their values in 01/1984

* Simulate the economy from 1984 and 2007 using the data on inflows and crop prices

\begin{tabular}{rrr}
\hline Source & Pricing Rule & Years of Water Shortage \\
\hline Data & Average-Cost Prices & $1989,1991,1994,1999,2001,2004,2005,2006$ \\
Model & Average-Cost Prices & $1989,1991,1994,1999,2001,2004,2006$ \\
Model & Optimal Prices & \\
\hline
\end{tabular}

Table: Water Shortages in the Turkish Data 


\section{Forecasts and Counterfactuals}

Implications of the Pricing Policies on Water Resource Management

- Under the current pricing policy (break-even prices), the government experiences water shortage every 8 years, with a standard deviation of 8 years.

- Under the current pricing policy (break-even prices), the government experiences a severe water shortage (below subsistence) every 50 years.

- If the government chooses the water prices optimally, water shortages never occur.

\begin{tabular}{rrrrrr}
\hline Source & Pricing Rule & Type & Mean Year & Std Year & Mean No \\
\hline Model & Optimal & Water Shortages & 100.000 & 0 & 0 \\
Model & Average-Cost & Water Shortages & 8.237 & 8.120 & 10.687 \\
Model & Average-Cost & Below Subsistence & 50.998 & 35.516 & 1.530 \\
\hline
\end{tabular}

Table: Comparison of Average-Cost and Optimal Pricing Rules 


\section{Counterfactuals}

Keeping the current pricing policy, what are some alternative methods to target so many years without water shortages?

- Efficiency of water usage in production

* A one percent increase in irrigation efficiency delays water shortages for 12 years, on average.

* A five percent increase in irrigation efficiency delays water shortages for 68 years, on average.

* How can this be implemented? Switching irrigation methods: from surface to drip/sprinkler irrigation technologies.

\begin{tabular}{rr}
\hline \multicolumn{2}{c}{ Improvements in Irrigation } \\
$\%$ Change & Years of No Water Shortage \\
\hline 1 & 12.108 \\
2 & 22.537 \\
3 & 41.719 \\
4 & 59.377 \\
5 & 68.884 \\
\hline
\end{tabular}

Table: Percent Improvement in Irrigation 


\section{Counterfactuals}

Keeping the current pricing policy, what are some alternative methods to target so many years without water shortages?

- Supply-side improvements

* A one $\mathrm{hm}^{3}$ increase in monthly inflows delays water shortages for 17 years, on average.

* A five $\mathrm{hm}^{3}$ increase in monthly efficiency delays water shortages for a century, on average.

* How can this be implemented? Preventing leakages.

\begin{tabular}{rrr}
\hline & \multicolumn{3}{l}{ Increase in Monthly Inflows } \\
$\mathrm{hm}^{3}$ Change & $\%$ Change & Years of No Water Shortage \\
\hline 1 & 2.346 & 17.363 \\
2 & 4.691 & 58.455 \\
3 & 7.037 & 78.138 \\
4 & 9.382 & 96.588 \\
5 & 11.728 & 99.810 \\
\hline
\end{tabular}

Table: Improvement in Mean Annual Inflows 


\section{Conclusion}

- Model fit:

* Under the current policy, I replicated the years of water shortages observed in the data, except for 2005.

* The government could have avoided these water shortages observed in the data under the optimal pricing rule.

- Any extensions? Sağlam (2012).

* Profits from supplying water can be saved for the next period.

* External water resource which can supply water, if desired, at a certain cost to avoid water shortages.

Desalination technology, network of reservoirs

* Effects of cross-subsidization (often in favor of agriculture)

* Welfare comparisons of different pricing policies and counterfactual exercises. 


\section{Figure: Effect of Reservoir Capacity}
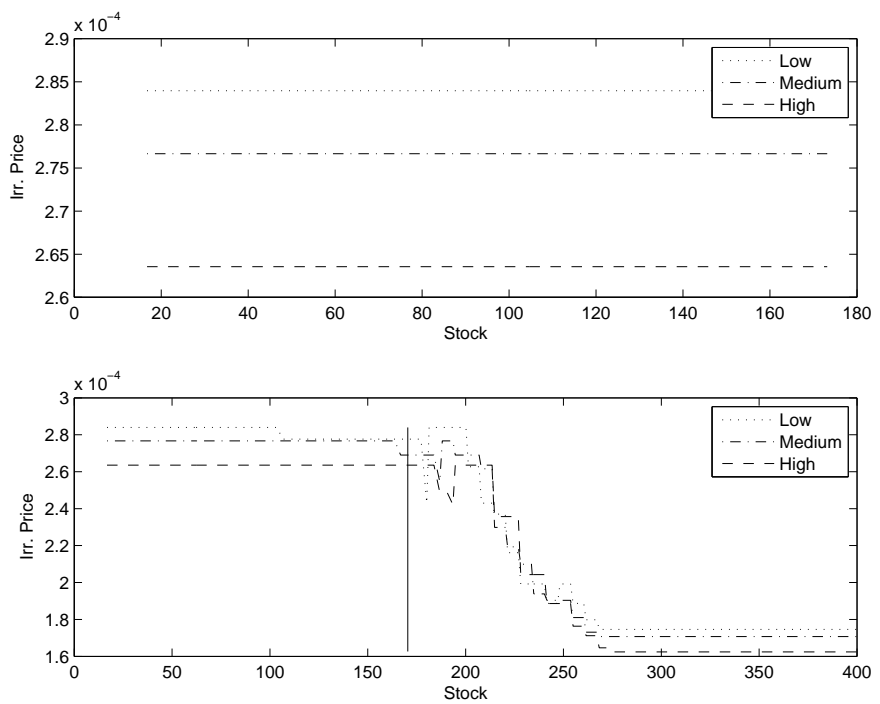

Figure: Policy Function for the Irrigation Price 


\section{Dynamic Ramsey Pricing}

The monthly value functions $m=0,1, \ldots, 11$ can be defined in the following way:

$$
\begin{aligned}
V(w, \mathbf{p}-1 ; \boldsymbol{\theta}, m)= & \max _{<w^{\prime}, W_{3}, \mathbf{p}>} U(\mathbf{p}, \boldsymbol{\tau} ; \boldsymbol{\theta}, m)+\beta \mathcal{E}\left[V\left(w^{\prime}, \mathbf{p} ; \boldsymbol{\theta}^{\prime}, m+1 \bmod 12\right)\right] \\
\ni & w^{\prime}=S(w, \boldsymbol{\theta})-\left\{W_{1}(\mathbf{p}, \boldsymbol{\tau} ; \boldsymbol{\theta}, m)+\mathcal{E}\left[W_{2}(\mathbf{p} ; \boldsymbol{\theta})\right] \delta_{m^{\prime}}^{m}+W_{3}\right\} \\
& \left\{\begin{array}{l}
\mathcal{E}\left[R_{i}(\mathbf{p}, \boldsymbol{\tau} ; \boldsymbol{\theta})\right]=\mathcal{E}\left[C_{i}(\mathbf{p}, \boldsymbol{\tau} ; \boldsymbol{\theta})\right]+\tau_{i} /(1-\lambda) ; \forall i=1,2 ; \text { if } m=0 \\
p_{i}=p_{i,-1} ; \forall i=1,2 ; \text { otherwise }
\end{array}\right. \\
& W_{1}(\mathbf{p}, \boldsymbol{\tau} ; \boldsymbol{\theta}, m), W_{2}(\mathbf{p} ; \boldsymbol{\theta}), W_{3}, \mathbf{p}, \boldsymbol{\tau} \geq \mathbf{0}
\end{aligned}
$$




\section{Estimation: Irrigation Water}

- Partial Equilibrium: shock to the profit function

- Observed profit function:

$$
\begin{aligned}
\Pi & =\max \left(\Pi_{1}, \ldots, \Pi_{N}, \Pi_{N+1}\right) \text { where } \\
\Pi_{c} & =\max _{<\ell_{c}>}\left(p_{f c} f\left(\ell_{c}, w_{2 c}\right)-p_{2} w_{2 c}+\mu_{c} \ell_{c}\right) ; \forall c=1, \ldots, N \\
\ni \ell_{c} & \leq \bar{\ell}=1, \\
\Pi_{N+1} & =0
\end{aligned}
$$

- Although farmers make discrete choices, the government only has a probability distribution over crops. 